\title{
Application of reproducing kernel Hilbert space method for solving second-order fuzzy Volterra integro-differential equations
}

\author{
Ghaleb N. Gumah', Mohammad F.M. Naser ${ }^{1}$, Mohammed Al-Smadi² and Shrideh K. Al-Omari ${ }^{1 *}$ (D)
}

"Correspondence:

s.k.q.alomari@fet.edu.jo

${ }^{1}$ Faculty of Engineering Technology,

Al-Balqa Applied University,

Amman, Jordan

Full list of author information is

available at the end of the article

\begin{abstract}
In this article, we propose a new method that determines an efficient numerical procedure for solving second-order fuzzy Volterra integro-differential equations in a Hilbert space. This method illustrates the ability of the reproducing kernel concept of the Hilbert space to approximate the solutions of second-order fuzzy Volterra integro-differential equations. Additionally, we discuss and derive the exact and approximate solutions in the form of Fourier series with effortlessly computable terms in the reproducing kernel Hilbert space $W_{2}^{3}[a, b] \oplus W_{2}^{3}[a, b]$. The convergence of the method is proven and its exactness is illustrated by three numerical examples.
\end{abstract}

Keywords: Reproducing kernel Hilbert space; Complete orthonormal system; Fuzzy Volterra integro-differential equation

\section{Introduction}

Fuzzy integro-differential equations are very useful tools for modeling physical systems under the differential sense and dynamical systems under the possibilistic uncertainty [1]. In recent years, fuzzy Volterra integro-differential equations (FVIDEs) in both theoretical and numerical calculations have been growing in many scientific applications [2-4]. They are usually encountered in many applications including fuzzy modeling in population dynamics, experimental quantum optics to quantum gravity, and averaging fuzzy biopolymers (see [5-7] and the references therein). Biswas et al. [8] have recently proposed a numerical fuzzy differential transform (FDT) method for solving second-order FVIDEs under the generalized concept of Seikkala differentiability of fuzzy functions. A further literature review of fuzzy integro-differential equations can be found in $[2,3]$ and [8].

The main goal of this article is to solve second-order FVIDEs in the Hilbert space $W_{2}^{3}[a, b] \oplus W_{2}^{3}[a, b]$ under the assumption of strongly generalized differentiability. More precisely, we provide a numerical approximate solution for fuzzy Volterra integrodifferential equation of the general form

$$
y^{\prime \prime}(t)=f(t)+\int_{a}^{t} k(t, s) g(y(s)) d s, \quad t \geq a,
$$

(c) The Author(s) 2018. This article is distributed under the terms of the Creative Commons Attribution 4.0 International License (http://creativecommons.org/licenses/by/4.0/), which permits unrestricted use, distribution, and reproduction in any medium, provided you give appropriate credit to the original author(s) and the source, provide a link to the Creative Commons license, and indicate if changes were made. 
subject to the fuzzy initial conditions

$$
y(a)=\gamma_{1}, \quad y^{\prime}(a)=\gamma_{2},
$$

where $k(s, t)$ is an arbitrary kernel function, $g$ is a continuous function, $f$ is a continuous fuzzy-valued function, and $\gamma_{1}, \gamma_{2}$ are fuzzy real numbers. In special cases the analytic solution of FVIDE (1)-(2) can be found using the fuzzy Laplace transform (FLT) method. However, a fuzzy solution of FVIDE (1)-(2) is usually very difficult to be derived analytically. To solve this issue, FVIDE (1)-(2) can be converted into two systems of Volterra integro-differential equations which are solvable numerically using the reproducing kernel Hilbert space (RKHS) method based on the Gram-Schmidt orthogonalization process. Moreover, the authors in [9] have proven the existence and uniqueness of a fuzzy solution for the second-order FVIDE (1)-(2) with the fuzzy kernel under strongly generalized differentiability.

Reproducing kernel theory has important scientific applications in numerical analysis, ordinary and fractional differential equations, probability, statistics, and learning theory. Recently, RKHS method for solving a variety of fuzzy differential equations and fuzzy fractional differential equations has been presented by Ahmadian et al. [10, 11]. The RKHS method possesses many virtues; to begin with, it is conceivable to choose any point in the interval of integration and as well the approximate solution would be appropriate and applicable. Secondly, it is precise and requires less exertion to discover the numerical results. Thirdly, it is a proficient application to solve some scientific models in comparison with the numerical solutions and the exact solutions. Fourthly, the method is easy to understand, and it needs little computational prerequisites. For more points of interest and descriptions about the RKHS method, we allude to [12-17].

Our paper has the following structure. Section 2 presents some concepts and results about the fuzzy numbers and the properties of fuzzy-number-valued functions, including the $H$-differentiability or Hukuhara differentiability concept and the fuzzy integral. The fuzzy Volterra integro-differential equations of the second order are introduced in Sect. 3. Section 4 proposes a numerical method for solving fuzzy Volterra integro-differential equations of second order. In Sect. 5, we present three numerical examples to illustrate our method in Sect. 4. Finally, we end the paper with the conclusion in Sect. 6.

\section{Preliminaries}

We recall some basic definitions and theorems needed throughout the paper such as fuzzy number, fuzzy-valued function, and the derivative of the fuzzy-valued functions.

Definition 2.1 (see [18]) A fuzzy number $u: \mathbb{R} \longrightarrow[0,1]$ is a fuzzy subset of $\mathbb{R}$ with normal, convex, and upper semicontinuous membership function of bounded support.

Let $R_{\digamma}$ denote the space of fuzzy real numbers. For $0<\alpha \leq 1$, set $[u]_{\alpha}=\{s \in \mathbb{R} \mid u(s) \geq \alpha\}$ and $[u]_{0}=\overline{\{s \in \mathbb{R} \mid u(s)>0\}}$ (the closure of $\{s \in \mathbb{R} \mid u(s)>0\}$ ). Then the $\alpha$-level set $[u]_{\alpha}$ is a nonempty compact interval for all $0 \leq \alpha \leq 1$ and any $u \in R_{\digamma}$. The notation $[u]_{\alpha}=$ $\left[u_{1}(\alpha), u_{2}(\alpha)\right]$ denotes explicitly the $\alpha$-level set of $u$. We refer to $u_{1}$ and $u_{2}$ as the lower and upper branches on $u$, respectively. 
Theorem 2.2 (see [19]) A mapping $u: \mathbb{R} \longrightarrow[0,1]$ is a fuzzy number with $\alpha$-cut representation $\left[u_{1}(\alpha), u_{2}(\alpha)\right]$ if and only if the following conditions are satisfied:

(i) the function $u_{1}:[0,1] \rightarrow \mathbb{R}$ is bounded nondecreasing;

(ii) the function $u_{2}:[0,1] \rightarrow \mathbb{R}$ is bounded nonincreasing;

(iii) for each $r \in(0,1], \lim _{\alpha \rightarrow r^{-}} u_{1}(\alpha)=u_{1}(r)$ and $\lim _{\alpha \rightarrow r^{-}} u_{2}(\alpha)=u_{2}(r)$;

(iv) for each $r \in(0,1], \lim _{\alpha \rightarrow r^{+}} u_{1}(\alpha)=u_{1}(r)$ and $\lim _{\alpha \rightarrow r^{+}} u_{2}(\alpha)=u_{2}(r)$;

(v) $u_{1}(\alpha) \leq u_{2}(\alpha)$ for all $\alpha \in[0,1]$.

For $u, v \in R_{\digamma}$ and $\lambda \in \mathbb{R}$, the sum $u+v$ and the product $\lambda u$ are defined by $[u+v]_{\alpha}=$ $[u]_{\alpha}+[v]_{\alpha},[\lambda u]_{\alpha}=\lambda[u]_{\alpha}, \forall \alpha \in[0,1]$, where $[u]_{\alpha}+[v]_{\alpha}$ means the usual addition of two intervals (subsets) of $\mathbb{R}$ and $\lambda[u]_{\alpha}$ is the usual product between a scalar and a subset of $\mathbb{R}$. The metric structure is given by the Hausdorff distance $D: R_{\digamma} \times R_{\digamma} \longrightarrow \mathbb{R}^{+} \cup\{0\}$, by $D(u, v)=\sup _{\alpha \in[0,1]} \max \left\{\left|u_{1}-v_{1}\right|,\left|u_{2}-v_{2}\right|\right\}$.

Definition 2.3 (see [18]) Let $y:[a, b] \longrightarrow R_{\digamma}$ and $t_{0} \in(a, b)$. We say $y$ is (1)-differentiable at $t_{0}$ if there is some element $y^{\prime}\left(t_{0}\right) \in R_{\digamma}$ such that, for all $h>0$ sufficiently close to 0 , there exist $y\left(t_{0}+h\right) \ominus y\left(t_{0}\right), y\left(t_{0}\right) \ominus y\left(t_{0}-h\right)$, and the limits (in the metric $D$ )

$$
\lim _{h \rightarrow 0^{+}} \frac{y\left(t_{0}+h\right) \ominus y\left(t_{0}\right)}{h}=\lim _{h \rightarrow 0^{+}} \frac{y\left(t_{0}\right) \ominus y\left(t_{0}-h\right)}{h}=y^{\prime}\left(t_{0}\right) .
$$

In this case, we denote $y^{\prime}\left(t_{0}\right)$ by $D_{1}^{1} y\left(t_{0}\right)$. Also, $y$ is (2)-differentiable if, for all $h<0$ sufficiently close to 0 , there exist $y\left(t_{0}+h\right) \ominus y\left(t_{0}\right), y\left(t_{0}\right) \ominus y\left(t_{0}-h\right)$, and the limits (in the metric $D$ )

$$
\lim _{h \rightarrow 0^{-}} \frac{y\left(t_{0}+h\right) \ominus y\left(t_{0}\right)}{h}=\lim _{h \rightarrow 0^{-}} \frac{y\left(t_{0}\right) \ominus y\left(t_{0}-h\right)}{h}=y^{\prime}\left(t_{0}\right) .
$$

In this case, this derivative is denoted by $D_{2}^{1} y\left(t_{0}\right)$.

Theorem 2.4 (see [20]) Let $y:[a, b] \longrightarrow R_{\digamma}$ be a fuzzy-valued function, where $[y(t)]_{\alpha}=$ $\left[y_{1, \alpha}(t), y_{2, \alpha}(t)\right]$ for each $\alpha \in[0,1]$.

(i) If $y$ is (1)-differentiable, then $y_{1, \alpha}$ and $y_{2, \alpha}$ are differentiable functions and $\left[D_{1}^{1} y(t)\right]_{\alpha}=\left[y_{1, \alpha}^{\prime}(t), y_{2, \alpha}^{\prime}(t)\right]$.

(ii) If $y$ is (2)-differentiable, then $y_{1, \alpha}$ and $y_{2, \alpha}$ are differentiable functions and $\left[D_{2}^{1} y(t)\right]_{\alpha}=\left[y_{2, \alpha}^{\prime}(t), y_{1, \alpha}^{\prime}(t)\right]$.

Theorem 2.5 (see [21]) Let $D_{1}^{1} y:[a, b] \longrightarrow R_{\digamma}$ or $D_{2}^{1} y:[a, b] \longrightarrow R_{\digamma}$ be fuzzy-valued functions, where $[y(t)]_{\alpha}=\left[y_{1, \alpha}(t), y_{2, \alpha}(t)\right]$ for each $\alpha \in[0,1]$.

(i) If $D_{1}^{1} y$ is (1)-differentiable, then $y_{1, \alpha}^{\prime}$ and $y_{2, \alpha}^{\prime}$ are differentiable functions and $\left[y^{\prime \prime}(t)\right]_{\alpha}=\left[y_{1, \alpha}^{\prime \prime}(t), y_{2, \alpha}^{\prime \prime}(t)\right]$.

(ii) If $D_{1}^{1} y$ is (2)-differentiable, then $y_{1, \alpha}^{\prime}$ and $y_{2, \alpha}^{\prime}$ are differentiable functions and $\left[y^{\prime \prime}(t)\right]_{\alpha}=\left[y_{2, \alpha}^{\prime \prime}(t), y_{1, \alpha}^{\prime \prime}(t)\right]$.

(iii) If $D_{2}^{1} y$ is (1)-differentiable, then $y_{1, \alpha}^{\prime}$ and $y_{2, \alpha}^{\prime}$ are differentiable functions and $\left[y^{\prime \prime}(t)\right]_{\alpha}=\left[y_{2, \alpha}^{\prime \prime}(t), y_{1, \alpha}^{\prime \prime}(t)\right]$.

(iv) If $D_{2}^{1} y$ is (2)-differentiable, then $y_{1, \alpha}^{\prime}$ and $y_{2, \alpha}^{\prime}$ are differentiable functions and $\left[y^{\prime \prime}(t)\right]_{\alpha}=\left[y_{1, \alpha}^{\prime \prime}(t), y_{2, \alpha}^{\prime \prime}(t)\right]$. 
Theorem 2.6 (see [22]) Let $y:[a, b] \longrightarrow R_{\digamma}$ be a continuous fuzzy function, where $[y(t)]_{\alpha}=$ $\left[y_{1, \alpha}(t), y_{2, \alpha}(t)\right]$. If $y_{1, \alpha}(t)$ and $y_{2, \alpha}(t)$ are integrable functions over $[a, b]$, then $\int_{a}^{b} y(t) d t \in R_{\digamma}$ and

$$
\left[\int_{a}^{b} y(t) d t\right]_{\alpha}=\left[\int_{a}^{b} y_{1, \alpha}(t) d t, \int_{a}^{b} y_{2, \alpha}(t) d t\right]
$$

\section{Formulation of second-order FVIDE}

This section studies the second-order FVIDEs under the concept of strongly generalized differentiability in which the FVIDE is converted into an equivalent system. This system consists of the crisp system of Volterra integro-differential equations for each type of differentiability.

In order to design a numerical scheme for solving FVIDE (1)-(2), we first replace it according to Theorem 2.5 by the following two systems:

$(1,1)$-system

$$
\begin{aligned}
& y_{1, \alpha}^{\prime \prime}(t)=f_{1, \alpha}(t)+\int_{a}^{t} \underline{U}(t, \alpha) d s, \\
& y_{2, \alpha}^{\prime \prime}(t)=f_{2, \alpha}(t)+\int_{a}^{t} \bar{U}(t, \alpha) d s ;
\end{aligned}
$$

$(1,2)$-system

$$
\begin{aligned}
& y_{2, \alpha}^{\prime \prime}(t)=f_{1, \alpha}(t)+\int_{a}^{t} \underline{U}(t, \alpha) d s, \\
& y_{1, \alpha}^{\prime \prime}(t)=f_{2, \alpha}(t)+\int_{a}^{t} \bar{U}(t, \alpha) d s .
\end{aligned}
$$

Depending on the Zadeh extension principle in [23], if $g$ in Eq. (1) is a strictly increasing function, then

$$
\underline{U}(t, \alpha)= \begin{cases}k(t, s) g\left(y_{1, \alpha}(s)\right), & k(t, s) \geq 0, \\ k(t, s) g\left(y_{2, \alpha}(s)\right), & k(t, s)<0,\end{cases}
$$

and

$$
\bar{U}(t, \alpha)= \begin{cases}k(t, s) g\left(y_{2, \alpha}(s)\right), & k(t, s) \geq 0, \\ k(t, s) g\left(y_{1, \alpha}(s)\right), & k(t, s)<0 .\end{cases}
$$

Moreover, if $g$ in Eq. (1) is a strictly decreasing function, then

$$
\underline{U}(t, \alpha)= \begin{cases}k(t, s) g\left(y_{2, \alpha}(s)\right), & k(t, s) \geq 0, \\ k(t, s) g\left(y_{1, \alpha}(s)\right), & k(t, s)<0,\end{cases}
$$

and

$$
\bar{U}(t, \alpha)= \begin{cases}k(t, s) g\left(y_{1, \alpha}(s)\right), & k(t, s) \geq 0, \\ k(t, s) g\left(y_{2, \alpha}(s)\right), & k(t, s)<0 .\end{cases}
$$


Here, we want to mention that the sufficient conditions for the existence of a unique solution to the second-order FVIDE (1)-(2) have been given in [9].

\section{Analysis of the method with its convergence}

In this section, we present a review of the notations and preliminary definitions of the RKHS theory. Additionally, we demonstrate how to solve FVIDE (1)-(2) using the RKHS method. Accordingly, we build orthonormal function systems of the space $W_{2}^{3}[0,1] \oplus$ $W_{2}^{3}[0,1]$ based on the Gram-Schmidt orthogonalization process.

Definition 4.1 (see [24]) Let $H$ be a Hilbert space. A function $K: \Omega \times \Omega \rightarrow \mathbb{R}$ is a reproducing kernel of $H$ if the following conditions are satisfied:

(i) for each $x \in \Omega, K(\cdot, x) \in H$;

(ii) for each $x \in \Omega$ and $\phi \in H,\langle\phi, K(\cdot, x)\rangle=\phi(x)$, which is called the reproducing property.

Definition 4.2 (see [24]) The Hilbert space $W_{2}^{m}[a, b]$ is defined as $W_{2}^{m}[a, b]=\{y(t) \mid y(t)$, $y^{\prime}(t), \ldots, y^{(m-1)}(t)$ are absolutely continuous , $y^{(m)}(t) \in L^{2}[a, b]$ and $y(a)=y^{\prime}(a)=\cdots=$ $y^{(m-1)}(a)=0$ whenever $\left.m \neq 1\right\}$. Whilst the inner product and the norm in $W_{2}^{m}[a, b]$ are defined by

$$
\left\langle y_{1}(t), y_{2}(t)\right\rangle_{W_{2}^{m}}=\sum_{i=0}^{m-1} y_{1}^{(i)}(a) y_{2}^{(i)}(a)+\int_{a}^{b} y_{1}^{(m)}(t) y_{2}^{(m)}(t) d t
$$

and $\left\|y_{1}(t)\right\|_{W_{2}^{m}}=\sqrt{\left\langle y_{1}(t), y_{1}(t)\right\rangle_{W_{2}^{m}}}$, where $y_{1}, y_{2} \in W_{2}^{m}[a, b]$.

Definition 4.3 The Hilbert space $W_{2}^{m}[a, b] \oplus W_{2}^{m}[a, b], m=1,2,3, \ldots, n$, can be defined as $W_{2}^{m}[a, b] \oplus W_{2}^{m}[a, b]=\left\{y=\left(y_{1}, y_{2}\right)^{T} \mid y_{1}, y_{2} \in W_{2}^{m}[a, b]\right\}$. Accordingly, the inner product and the norm in $W_{2}^{m}[a, b] \oplus W_{2}^{m}[a, b]$ are built, respectively, by $\langle y(t), z(t)\rangle_{W_{2}^{m} \oplus W_{2}^{m}}=$ $\sum_{i=1}^{2}\left\langle y_{i}(t), z_{i}(t)\right\rangle_{W_{2}^{m}}$ and $\|y(t)\|_{W_{2}^{m} \oplus W_{2}^{m}}=\sqrt{\sum_{i=1}^{2}\left\|y_{i}(t)\right\|_{W_{2}^{m}}^{2}}$.

Theorem 4.4 (see [25]) The space $W_{2}^{m}[a, b]$ is a complete reproducing kernel space. That is, for each fixed $t \in[a, b]$, there exists $R_{t}(s) \in W_{2}^{m}[a, b]$ such that $\left\langle y(s), R_{t}(s)\right\rangle_{W_{2}^{m}}=y(t)$ for any $y(t) \in W_{2}^{m}[a, b]$ and any $t \in[a, b]$. The reproducing kernel $R_{t}(s)$ can be written as

$$
R_{t}(s)= \begin{cases}\sum_{i=0}^{2 m-1} p_{i}(t) s^{i}, & s \leq t, \\ \sum_{i=0}^{2 m-1} q_{i}(t) s^{i}, & s>t .\end{cases}
$$

The representation of the reproducing kernel function $R_{t}(s)$ in $W_{2}^{3}[0,1]$, using Mathematica 7.0 software package, is provided by

$$
R_{t}(s)= \begin{cases}1+\frac{1}{12} t^{2} s^{2}(3+t)+t s\left(1-\frac{1}{24} t^{4}\right)+\frac{1}{120} t^{5}, & s \leq t, \\ 1+\frac{1}{12} t^{2} s^{2}(3+s)+t s\left(1-\frac{1}{24} s^{4}\right)+\frac{1}{120} s^{5}, & s>t .\end{cases}
$$

To apply our technique on the Hilbert space $W_{2}^{3}[a, b] \oplus W_{2}^{3}[a, b]$, we define the linear invertible operator $L: W_{2}^{3}[a, b] \oplus W_{2}^{3}[a, b] \longrightarrow W_{2}^{1}[a, b] \oplus W_{2}^{1}[a, b]$ as $L y(t)=y^{\prime \prime}(t)$ such 
that $L=\left[\begin{array}{cc}L_{1} & 0 \\ 0 & L_{2}\end{array}\right]$ and $y(t)=\left(y_{1, \alpha}(t), y_{2, \alpha}(t)\right)^{T}$. Thus, Eqs. (1) and (2) can be converted into the form

$$
L y(t)=G(t, f(t), h(y(t)))
$$

subject to the fuzzy initial conditions

$$
y(a)=\gamma_{1}, \quad y^{\prime}(a)=\gamma_{2},
$$

where $h(y(t))=\int_{a}^{t} k(t, s) g(y(t)) d s, y(t) \in W_{2}^{3}[a, b] \oplus W_{2}^{3}[a, b]$, and $f(t) \in W_{2}^{1}[a, b] \oplus$ $W_{2}^{1}[a, b]$. Here, $G(t, f(t), h(y(t)))$ is $G\left(t, f_{1, \alpha}(t), f_{2, \alpha}(t), h\left(y_{1, \alpha}(t)\right), h\left(y_{2, \alpha}(t)\right)\right)$.

Lemma 4.5 $L: W_{2}^{3}[a, b] \oplus W_{2}^{3}[a, b] \longrightarrow W_{2}^{1}[a, b] \oplus W_{2}^{1}[a, b]$ is a bounded linear operator.

Proof Firstly, it is easy to prove that $L$ is a linear operator from $W_{2}^{3}[a, b] \oplus W_{2}^{3}[a, b]$ into $W_{2}^{1}[a, b] \oplus W_{2}^{1}[a, b]$. Secondly, we need to prove that $\|L y\|_{W_{2}^{1} \oplus W_{2}^{1}} \leq \xi\|y\|_{W_{2}^{3} \oplus W_{2}^{3}}$, where $\xi>0$. For each $y \in W_{2}^{3}[a, b] \oplus W_{2}^{3}[a, b]$, we have

$$
\begin{aligned}
\|L y(t)\|_{W_{2}^{1} \oplus W_{2}^{1}}^{2}= & \sum_{i=1}^{2}\left\|L_{i} y_{i, \alpha}(t)\right\|_{W_{2}^{1}}^{2}=\left\|L_{1} y_{1, \alpha}(t)\right\|_{W_{2}^{1}}^{2}+\left\|L_{2} y_{2, \alpha}(t)\right\|_{W_{2}^{1}}^{2} \\
= & \left\langle L_{1} y_{1, \alpha}(t), L_{1} y_{1, \alpha}(t)\right\rangle_{W_{2}^{1}}+\left\langle L_{2} y_{2, \alpha}(t), L_{2} y_{2, \alpha}(t)\right\rangle_{W_{2}^{1}} \\
= & {\left[L_{1} y_{1, \alpha}(a)\right]^{2}+\int_{a}^{b}\left[\frac{d}{d t}\left(L_{1} y_{1, \alpha}(t)\right)\right]^{2} d t+\left[L_{2} y_{2, \alpha}(a)\right]^{2} } \\
& +\int_{a}^{b}\left[\frac{d}{d t}\left(L_{2} y_{2, \alpha}(t)\right)\right]^{2} d t .
\end{aligned}
$$

By the reproducing property of $R_{t}(s)$, then $y_{1, \alpha}(t)=\left\langle y_{1, \alpha}(s), R_{t}(s)\right\rangle_{W_{2}^{3}}$ and $y_{2, \alpha}(t)=\left\langle y_{2, \alpha}(s)\right.$, $\left.R_{t}(s)\right\rangle_{W_{2}^{3}}$. Thus,

$$
\begin{aligned}
& L_{1} y_{1, \alpha}(t)=\left\langle y_{1, \alpha}(s), L_{1} R_{t}(s)\right\rangle_{W_{2}^{3}}, \quad L_{2} y_{2, \alpha}(t)=\left\langle y_{2, \alpha}(s), L_{2} R_{t}(s)\right\rangle_{W_{2}^{3}}, \\
& \frac{d}{d t}\left(L_{1} y_{1, \alpha}(t)\right)=\left\langle y_{1, \alpha}(s), \frac{d}{d t}\left(L_{1} R_{t}(s)\right)\right\rangle_{W_{2}^{3}} \\
& \frac{d}{d t}\left(L_{2} y_{2, \alpha}(t)\right)=\left\langle y_{2, \alpha}(s), \frac{d}{d t}\left(L_{2} R_{t}(s)\right)\right\rangle_{W_{2}^{3}} .
\end{aligned}
$$

From the continuity of $R_{t}(s)$ on $[a, b]$, we have

$$
\begin{aligned}
& \left|L_{1} y_{1, \alpha}(t)\right|=\left|\left\langle y_{1, \alpha}(s), L_{1} R_{t}(s)\right\rangle_{W_{2}^{3}}\right| \leq\left\|y_{1, \alpha}\right\|_{W_{2}^{3}}\left\|L_{1} R_{t}(s)\right\|_{W_{2}^{3}} \leq k_{1}\left\|y_{1, \alpha}\right\|_{W_{2}^{3}}, \\
& \left|L_{2} y_{2, \alpha}(t)\right|=\left|\left\langle y_{2, \alpha}(s), L_{2} R_{t}(s)\right\rangle_{W_{2}^{3}}\right| \leq\left\|y_{2, \alpha}\right\|_{W_{2}^{3}}\left\|L_{2} R_{t}(s)\right\|_{W_{2}^{3}} \leq k_{2}\left\|y_{2, \alpha}\right\|_{W_{2}^{3}}, \\
& \left|\frac{d}{d t}\left(L_{1} y_{1, \alpha}(t)\right)\right|=\left|\left\langle y_{1, \alpha}(s), \frac{d}{d t}\left(L_{1} R_{t}(s)\right)\right\rangle_{W_{2}^{3}}\right| \leq\left\|y_{1, \alpha}\right\|_{W_{2}^{3}}\left\|\frac{d}{d t}\left(L_{1} R_{t}(s)\right)\right\|_{W_{2}^{3}} \\
& \leq k_{3}\left\|y_{1, \alpha}\right\|_{W_{2}^{3}},
\end{aligned}
$$




$$
\begin{aligned}
\left|\frac{d}{d t}\left(L_{2} y_{2, \alpha}(t)\right)\right| & =\left|\left\langle y_{2, \alpha}(s), \frac{d}{d t}\left(L_{2} R_{t}(s)\right)\right\rangle_{W_{2}^{3}}\right| \leq\left\|y_{2, \alpha}\right\|_{W_{2}^{3}}\left\|\frac{d}{d t}\left(L_{2} R_{t}(s)\right)\right\|_{W_{2}^{3}} \\
& \leq k_{4}\left\|y_{2, \alpha}\right\|_{W_{2}^{3}} .
\end{aligned}
$$

Thus,

$$
\begin{aligned}
\|L y(t)\|_{W_{2}^{1} \oplus W_{2}^{1}}^{2} & \leq k_{1}^{2}\left\|y_{1, \alpha}\right\|_{W_{2}^{3}}^{2}+(b-a) k_{3}^{2}\left\|y_{1, \alpha}\right\|_{W_{2}^{3}}^{2}+k_{2}^{2}\left\|y_{2, \alpha}\right\|_{W_{2}^{3}}^{2}+(b-a) k_{4}^{2}\left\|y_{2, \alpha}\right\|_{W_{2}^{3}}^{2} \\
& =\left(k_{1}^{2}+(b-a) k_{3}^{2}\right)\left\|y_{1, \alpha}\right\|_{W_{2}^{3}}^{2}+\left(k_{2}^{2}+(b-a) k_{4}^{2}\right)\left\|y_{2, \alpha}\right\|_{W_{2}^{3}}^{2} \\
& \leq \xi^{2}\left(\left\|y_{1, \alpha}\right\|_{W_{2}^{3}}^{2}+\left\|y_{2, \alpha}\right\|_{W_{2}^{3}}^{2}\right) \\
& =\xi^{2} \sum_{i=1}^{2}\left\|y_{i, \alpha}\right\|_{W_{2}^{3}}^{2} \\
& =\xi^{2}\|y\|_{W_{2}^{3} \oplus W_{2}^{3}}^{2},
\end{aligned}
$$

where $\xi^{2}=\max \left\{k_{1}^{2}+(b-a) k_{3}^{2}, k_{2}^{2}+(b-a) k_{4}^{2}\right\}$. The proof is complete.

Next, to construct an orthogonal system of functions $\left\{\psi_{i j}(t)\right\}_{(i, j)=(1,1)}^{(\infty, 2)}$ of the space $W_{2}^{3}[a, b] \oplus W_{2}^{3}[a, b]$, we let $\psi_{i j}(t)=L^{*} \Phi_{i j}(t)$, where $L^{*}=\left[\begin{array}{cc}L_{1}^{*} & 0 \\ 0 & L_{2}^{*}\end{array}\right]$ is the adjoint operator of $L$ and $\Phi_{i j}(t)=\left(\Phi_{i 1}(t), \Phi_{i 2}(t)\right)^{T}$. From now on the orthonormal system $\left\{\bar{\psi}_{i j}(t)\right\}_{(i, j)=(1,1)}^{(\infty, 2)}$ in the space $W_{2}^{3}[a, b] \oplus W_{2}^{3}[a, b]$ can be derived from the Gram-Schmidt orthogonalization process of $\left\{\psi_{i j}(t)\right\}_{(i, j)=(1,1)}^{(\infty, 2)}$ as follows:

$$
\bar{\psi}_{i j}(t)=\sum_{l=1}^{i} \sum_{k=1}^{j} \beta_{l k}^{i j} \psi_{l k}(t)
$$

where $\beta_{l k}^{i j}$ are orthogonalization coefficients given by

$$
\begin{aligned}
& \beta_{11}^{i j}=\frac{1}{\left\|\psi_{11}\right\|}, \quad \beta_{l k}^{i j}=\left(\left\|\psi_{l k}\right\|^{2}-\sum_{p=1}^{l-1}\left\langle\psi_{l k}(t), \bar{\psi}_{l p}(t)\right\rangle^{2}\right)^{-\frac{1}{2}} \quad(l=k \neq 1), \\
& \beta_{l k}^{i j}=\frac{-\sum_{p=k}^{l-1}\left\langle\psi_{l k}(t), \bar{\psi}_{l p}(t)\right\rangle \beta_{p k}^{i j}}{\sqrt{\left\|\psi_{l k}\right\|^{2}-\sum_{p=1}^{l-1}\left\langle\psi_{l k}(t), \bar{\psi}_{l p}(t)\right\rangle^{2}}} \quad(l>k) .
\end{aligned}
$$

The main result of this article is the following theorems, which give the exact expression of the solution of FVIDE (7)-(8) in the space $W_{2}^{3}[a, b] \oplus W_{2}^{3}[a, b]$ and the convergence of our method.

Theorem 4.6 Suppose $\left\{t_{i}\right\}_{i=1}^{\infty}$ is dense in $[a, b]$, if $y(t) \in W_{2}^{3}[a, b] \oplus W_{2}^{3}[a, b]$ is the solution of FVIDE (7)-(8), then

$$
y(t)=\sum_{i=1}^{\infty} \sum_{j=1}^{2} \sum_{l=1}^{i} \sum_{k=1}^{j} \beta_{l k}^{i j} G_{k}\left(t_{l}, f\left(t_{l}\right), h\left(y\left(t_{l}\right)\right)\right) \bar{\psi}_{i j}(t),
$$

which is a convergent series in the sense of $\|\cdot\|_{W_{2}^{3} \oplus W_{2}^{3}}$. 
Proof Firstly, we need to prove that $\left\{\psi_{i j}(t)\right\}_{(i, j)=(1,1)}^{(\infty, 2)}$ is the complete system in $W_{2}^{3}[a, b] \oplus$ $W_{2}^{3}[a, b]$ as follows:

$$
\begin{aligned}
\psi_{i j}(t) & =L^{*} \Phi_{i j}(t)=\left\langle L^{*} \Phi_{i j}(s), R_{t}(s)\right\rangle_{W_{2}^{3} \oplus W_{2}^{3}} \\
& =\left\langle\Phi_{i j}(s), L_{s} R_{t}(s)\right\rangle_{W_{2}^{1} \oplus W_{2}^{1}} \\
& =\left.L_{s} R_{t}(s)\right|_{s=t_{i}} \in W_{2}^{3}[a, b] \oplus W_{2}^{3}[a, b] .
\end{aligned}
$$

On the other hand, for each $y \in W_{2}^{3}[a, b] \oplus W_{2}^{3}[a, b]$, let $\left\langle y(t), \psi_{i j}(t)\right\rangle_{W_{2}^{3} \oplus W_{2}^{3}}=0$. One has

$$
\begin{aligned}
\left\langle y(t), \psi_{i j}(t)\right\rangle_{W_{2}^{3} \oplus W_{2}^{3}} & =\left\langle y_{1, \alpha}(t), \psi_{i 1}(t)\right\rangle_{W_{2}^{3}}+\left\langle y_{2, \alpha}(t), \psi_{i 2}(t)\right\rangle_{W_{2}^{3}} \\
& =\left\langle y_{1, \alpha}(t), L_{1}^{*} \Phi_{i 1}(t)\right\rangle_{W_{2}^{3}}+\left\langle y_{2, \alpha}(t), L_{2}^{*} \Phi_{i 2}(t)\right\rangle_{W_{2}^{3}} \\
& =\left\langle L_{1} y_{1, \alpha}(t), \Phi_{i 1}(t)\right\rangle_{W_{2}^{1}}+\left\langle L_{2} y_{2, \alpha}(t), \Phi_{i 2}(t)\right\rangle_{W_{2}^{1}} \\
& =L_{1} y_{1, \alpha}\left(t_{i}\right)+L_{2} y_{2, \alpha}\left(t_{i}\right) \\
& =L y\left(t_{i}\right) .
\end{aligned}
$$

Thus, $L y(t)=0$ because $\left\{t_{i}\right\}_{i=1}^{\infty}$ is dense on $[a, b]$. Hence, $y(t)=0$ because of the existence of $L^{-1}$. Secondly, since $\left\{\psi_{i j}(t)\right\}_{(i, j)=(1,1)}^{(\infty, 2)}$ is the complete system in $W_{2}^{3}[a, b] \oplus W_{2}^{3}[a, b]$ and from Eq. (9), then the sequence $\left\{\bar{\psi}_{i j}(t)\right\}_{(i, j)=(1,1)}^{(\infty, 2)}$ is the complete orthonormal system in $W_{2}^{3}[a, b] \oplus$ $W_{2}^{3}[a, b]$. Thirdly, using Fourier series expansion about $\left\{\bar{\psi}_{i j}(t)\right\}_{(i, j)=(1,1)}^{(\infty, 2)}$, we have

$$
\begin{aligned}
y(t) & =\sum_{i=1}^{\infty} \sum_{j=1}^{2}\left\langle y(t), \bar{\psi}_{i j}(t)\right\rangle_{W_{2}^{3} \oplus W_{2}^{3}} \bar{\psi}_{i j}(t) \\
& =\sum_{i=1}^{\infty} \sum_{j=1}^{2}\left\langle y(t), \sum_{l=1}^{i} \sum_{k=1}^{j} \beta_{l k}^{i j} \psi_{l k}(t)\right\rangle_{W_{2}^{3} \oplus W_{2}^{3}} \bar{\psi}_{i j}(t) \\
& =\sum_{i=1}^{\infty} \sum_{j=1}^{2} \sum_{l=1}^{i} \sum_{k=1}^{j} \beta_{l k}^{i j}\left\langle y(t), \psi_{l k}(t)\right\rangle_{W_{2}^{3} \oplus W_{2}^{3}} \bar{\psi}_{i j}(t) \\
& =\sum_{i=1}^{\infty} \sum_{j=1}^{2} \sum_{l=1}^{i} \sum_{k=1}^{j} \beta_{l k}^{i j}\left\langle y(t), L^{*} \Phi_{l k}(t)\right\rangle_{W_{2}^{3} \oplus W_{2}^{3}} \bar{\psi}_{i j}(t) \\
& =\sum_{i=1}^{\infty} \sum_{j=1}^{2} \sum_{l=1}^{i} \sum_{k=1}^{j} \beta_{l k}^{i j}\left\langle L y(t), \Phi_{l k}(t)\right\rangle_{W_{2}^{1} \oplus W_{2}^{1}} \bar{\psi}_{i j}(t) \\
& =\sum_{i=1}^{\infty} \sum_{j=1}^{2} \sum_{l=1}^{i} \sum_{k=1}^{j} \beta_{l k}^{i j}\left\langle G_{k}(t, f(t), h(y(t))), \Phi_{l k}(t)\right\rangle_{W_{2}^{1} \oplus W_{2}^{1}} \bar{\psi}_{i j}(t) \\
& =\sum_{i=1}^{\infty} \sum_{j=1}^{2} \sum_{l=1}^{i} \sum_{k=1}^{j} \beta_{l k}^{i j} G_{k}\left(t_{l}, f\left(t_{l}\right), h\left(y\left(t_{l}\right)\right)\right) \bar{\psi}_{i j}(t) .
\end{aligned}
$$


Since $\sum_{i=1}^{\infty} \sum_{j=1}^{2} \sum_{l=1}^{i} \sum_{k=1}^{j} \beta_{l k}^{i j} G_{k}\left(t_{l}, f\left(t_{l}\right), h\left(y\left(t_{l}\right)\right)\right) \bar{\psi}_{i j}(t)$ is a Fourier series in $W_{2}^{3}[a, b] \oplus$ $W_{2}^{3}[a, b]$ and $W_{2}^{3}[a, b] \oplus W_{2}^{3}[a, b]$ is a Hilbert space, then it is a convergent series in the

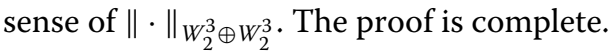

For numerical computations, we put the initial function $y_{0}\left(t_{l}\right)=y\left(t_{l}\right)$ and define the $n$ term numerical solution of FVIDE (7)-(8) by truncating the series given in Eq. (10) as follows:

$$
y_{n}(t)=\sum_{i=1}^{n} \sum_{j=1}^{2} \sum_{l=1}^{i} \sum_{k=1}^{j} \beta_{l k}^{i j} G_{k}\left(t_{l}, f\left(t_{l}\right), h\left(y_{l-1}\left(t_{l}\right)\right)\right) \bar{\psi}_{i j}(t) .
$$

Theorem 4.7 Suppose that $y(t)$ is the solution of FVIDE (7)-(8) and $y_{n}(t)$ is the approximate solution of FVIDE (7)-(8), where $y(t)$ and $y_{n}(t)$ are given by Eqs. (10) and (11), respectively, then

(i) if $\left\|y_{n}\right\|_{W_{2}^{3} \oplus W_{2}^{3}}$ is bounded and $\left\{t_{i}\right\}_{i=1}^{n}$ is dense on $[a, b]$, then $\left\|y_{n}-y\right\|_{W_{2}^{3} \oplus W_{2}^{3}} \longrightarrow 0$ as $n \rightarrow \infty$

(ii) $\left\|y_{n}-y\right\|_{C} \longrightarrow 0$ as $n \rightarrow \infty$;

(iii) the second derivative of $y_{n}$ converges to the second derivative of $y$ uniformly as $n \rightarrow \infty$;

(iv) if $\left\|y_{n-1}-y\right\|_{W_{2}^{3} \oplus W_{2}^{3}} \longrightarrow 0$ as $n \rightarrow \infty,\left\|y_{n-1}\right\|_{W_{2}^{3} \oplus W_{2}^{3}}$ is bounded, $t_{n} \rightarrow \tau$ as $n \rightarrow \infty$, $G(t, f(t), h(y(t)))$ is continuous for $t \in[a, b]$ and $f, h$ are continuous functions, then $G\left(t_{n}, f\left(t_{n}\right), h\left(y_{n-1}\left(t_{n}\right)\right)\right) \rightarrow G(\tau, f(\tau), h(y(\tau)))$.

Proof (i) From Eq. (11) and the orthonormality of $\left\{\bar{\psi}_{i j}(t)\right\}_{(i, j)=(1,1)}^{(\infty, 2)}$, we have $\left\|y_{n+1}\right\|_{W_{2}^{3} \oplus W_{2}^{3}}^{2}=$ $\left\|y_{0}\right\|_{W_{2}^{3} \oplus W_{2}^{3}}^{2}+\sum_{i=1}^{n+1} \sum_{j=1}^{2} A_{i j}^{2}$, where $A_{i j}=\sum_{l=1}^{i} \sum_{k=1}^{j} \beta_{l k}^{i j} G_{k}\left(t_{l}, f\left(t_{l}\right), h\left(y_{l-1}\left(t_{l}\right)\right)\right) \bar{\psi}_{i j}(t)$. Since $\left\|y_{n+1}\right\|_{W_{2}^{3} \oplus W_{2}^{3}} \geq\left\|y_{n}\right\|_{W_{2}^{3} \oplus W_{2}^{3}}$ and $\left\|y_{n}\right\|_{W_{2}^{3} \oplus W_{2}^{3}}$ is bounded, then $\left\|y_{n}\right\|_{W_{2}^{3} \oplus W_{2}^{3}}$ is convergent and $\left\{\sum_{j=1}^{2} A_{i j}^{2}\right\}_{i=1}^{\infty} \in l^{2}$. If $m>n$, then $\left\|y_{m}-y_{n}\right\|_{W_{2}^{3} \oplus W_{2}^{3}}^{2}=\sum_{l=n+1}^{m} \sum_{j=1}^{2} A_{i j}^{2} \rightarrow 0$ as $m, n \rightarrow \infty$. But $W_{2}^{3}[a, b] \oplus W_{2}^{3}[a, b]$ is a Hilbert space, then $\left\|y_{n}-y\right\|_{W_{2}^{3} \oplus W_{2}^{3}} \longrightarrow 0$ as $n \rightarrow \infty$. More details can be found in [26].

(ii) Based on the reproducing property of $R_{t}(s)$ and from (i), then

$$
\begin{aligned}
\left|y_{n}(t)-y(t)\right| & =\mid\left\langle y_{n}(s)-y(s),\left.R_{t}(s)\right|_{W_{2}^{3} \oplus W_{2}^{3}}\right| \\
& \leq\left\|y_{n}(s)-y(s)\right\|_{W_{2}^{3} \oplus W_{2}^{3}}\left\|\left(R_{t}(s)\right)\right\|_{W_{2}^{3} \oplus W_{2}^{3}} \\
& \leq k_{5}\left\|y_{n}-y\right\|_{W_{2}^{3} \oplus W_{2}^{3} \rightarrow 0 \quad \text { as } n \rightarrow \infty,}
\end{aligned}
$$

which means that $\left\|y_{n}-y\right\|_{C} \longrightarrow 0$ as $n \rightarrow \infty$.

(iii) Since $y_{n}$ converges to $y$ uniformly, then

$$
\begin{aligned}
\left|y_{n}^{\prime \prime}(t)-y^{\prime \prime}(t)\right| & =\left|\frac{d^{2}}{d t^{2}}\left(y_{n}(t)-y(t)\right)\right| \\
& =\left|\frac{d^{2}}{d t^{2}}\left\langle y_{n}(s)-y(s), R_{t}(s)\right\rangle_{W_{2}^{3} \oplus W_{2}^{3} \mid}\right| \\
& =\left|\left\langle y_{n}(s)-y(s), \frac{d^{2}}{d t^{2}}\left(R_{t}(s)\right)\right\rangle_{W_{2}^{3} \oplus W_{2}^{3} \mid}\right|
\end{aligned}
$$




$$
\begin{aligned}
& \leq\left\|y_{n}(s)-y(s)\right\|_{W_{2}^{3} \oplus W_{2}^{3}}\left\|\frac{d^{2}}{d t^{2}}\left(R_{t}(s)\right)\right\|_{W_{2}^{3} \oplus W_{2}^{3}} \\
& \leq k_{6}\left\|y_{n}-y\right\|_{W_{2}^{3} \oplus W_{2}^{3}} \rightarrow 0 \quad \text { as } n \rightarrow \infty .
\end{aligned}
$$

Thus, $\left\|y_{n}^{\prime \prime}-y^{\prime \prime}\right\|_{C} \rightarrow 0$ as $n \rightarrow \infty$.

(iv) Firstly, we prove that $y_{n-1}\left(t_{n}\right) \rightarrow y(\tau)(n \rightarrow \infty)$ as follows:

$$
\begin{aligned}
\left|y_{n-1}\left(t_{n}\right)-y(\tau)\right| & =\left|y_{n-1}\left(t_{n}\right)-y_{n-1}(\tau)+y_{n-1}(\tau)-y(\tau)\right| \\
& \leq\left|y_{n-1}\left(t_{n}\right)-y_{n-1}(\tau)\right|+\left|y_{n-1}(\tau)-y(\tau)\right| .
\end{aligned}
$$

From $\left\|y_{n-1}-y\right\|_{W_{2}^{3} \oplus W_{2}^{3}} \longrightarrow 0$ as $n \rightarrow \infty$, it follows that $\left|y_{n-1}(\tau)-y(\tau)\right| \rightarrow 0$ as $n \rightarrow \infty$. Based on the reproducing property of $R_{t}(s)$, then

$$
\begin{aligned}
\left|y_{n-1}\left(t_{n}\right)-y_{n-1}(\tau)\right| & =\left|\left\langle y_{n-1}(t), R_{t_{n}}(s)\right\rangle-\left\langle y_{n-1}(t), R_{\tau}(s)\right\rangle\right| \\
& =\left|\left\langle y_{n-1}(t), R_{t_{n}}(s)-R_{\tau}(s)\right\rangle\right| \\
& \leq\left\|y_{n-1}(t)\right\|_{W_{2}^{3} \oplus W_{2}^{3}}\left\|R_{t_{n}}(s)-R_{\tau}(s)\right\|_{W_{2}^{3} \oplus W_{2}^{3}} .
\end{aligned}
$$

Since the kernel function $R_{t}(s)$ is symmetric, $\left\|R_{t_{n}}(s)-R_{\tau}(s)\right\|_{W_{2}^{3} \oplus W_{2}^{3}} \rightarrow 0$ as $n \rightarrow \infty$, which means that $\left|y_{n-1}\left(t_{n}\right)-y_{n-1}(\tau)\right| \rightarrow 0$ as $n \rightarrow \infty$. Thus, $\left|y_{n-1}\left(t_{n}\right)-y(\tau)\right| \rightarrow 0$ as $n \rightarrow \infty$. Secondly, since $f$ and $h$ are continuous functions and $t_{n} \rightarrow \tau$, then $f\left(t_{n}\right) \rightarrow$ $f(\tau)$ as $n \rightarrow \infty$ and $h\left(y_{n-1}\left(t_{n}\right)\right) \rightarrow h(y(\tau))$ as $n \rightarrow \infty$. From the continuity of $G$, then $G\left(t_{n}, f\left(t_{n}\right), h\left(y_{n-1}\left(t_{n}\right)\right)\right) \rightarrow G(\tau, f(\tau), h(y(\tau)))$. The proof is complete.

\section{Numerical results}

In order to illustrate the accuracy of the method proposed in Sect. 4, we present three examples using Mathematica 7.0. The results obtained by our method are compared with the exact solution of each example and are found to be in good agreement with each other.

Example 5.1 Consider the following second-order FVIDE:

$$
y^{\prime \prime}(t)=[\alpha-1,1-\alpha]+\int_{0}^{t} y(s) d s, \quad 0 \leq t \leq 1, \alpha \in[0,1]
$$

subject to the fuzzy initial conditions

$$
y(0)=[\alpha-1,1-\alpha], \quad y^{\prime}(0)=[0,0] .
$$

Equations (12) and (13) are replaced by two systems of Volterra integro-differential equations as $(1,1)$-system

$$
\left.\begin{array}{l}
y_{1, \alpha}^{\prime \prime}(t)=(\alpha-1)+\int_{0}^{t} y_{1, \alpha}(s) d s, \quad 0 \leq t \leq 1, \\
y_{2, \alpha}^{\prime \prime}(t)=(1-\alpha)+\int_{0}^{t} y_{2, \alpha}(s) d s, \quad 0 \leq t \leq 1, \\
y_{1, \alpha}(0)=\alpha-1, \quad y_{2, \alpha}(0)=1-\alpha, \\
y_{1, \alpha}^{\prime}(0)=0, \quad y_{2, \alpha}^{\prime}(0)=0 .
\end{array}\right\}
$$


Table 1 Numerical results for $(1,1)$-system at $t=0.5$ and various $\alpha$

\begin{tabular}{lll}
\hline$\alpha$ & Exact solution & Approximate solution \\
\hline 0 & {$[-0.890245911500779,0.890245911500779]$} & {$[-0.888487875540245,0.888487875540245]$} \\
0.2 & {$[-0.712196729200623,0.712196729200623]$} & {$[-0.710790300432196,0.710790300432196]$} \\
0.4 & {$[-0.534147546900467,0.534147546900467]$} & {$[-0.533092725324147,0.533092725324147]$} \\
0.6 & {$[-0.356098364600311,0.356098364600311]$} & {$[-0.355395150216098,0.355395150216098]$} \\
0.8 & {$[-0.178049182300155,0.178049182300155]$} & {$[-0.177697575108049,0.177697575108049]$} \\
1 & {$[0,0]$} & {$[0,0]$} \\
\hline
\end{tabular}

Table 2 Numerical results for (1,2)-system at $t=0.5$ and various $\alpha$

\begin{tabular}{lll}
\hline$\alpha$ & Exact solution & Approximate solution \\
\hline 0 & {$[-0.8544486824714743,0.8544486824714743]$} & {$[-0.8521702477360533,0.8521702477360531]$} \\
0.2 & {$[-0.6835589459771796,0.6835589459771796]$} & {$[-0.6817361981888437,0.681736198188843]$} \\
0.4 & {$[-0.5126692094828845,0.5126692094828845]$} & {$[-0.5113021486416323,0.5113021486416318]$} \\
0.6 & {$[-0.3417794729885898,0.34177947298858898]$} & {$[-0.340868099909442184,0.3408680990944215]$} \\
0.8 & {$[-0.1708897364942948,0.1708897364942948]$} & {$[-0.17043404954721061,0.1704340495472106]$} \\
1 & {$[0,0]$} & {$[0,0]$} \\
\hline
\end{tabular}

$(1,2)$-system

$$
\left.\begin{array}{l}
y_{1, \alpha}^{\prime \prime}(t)=(1-\alpha)+\int_{0}^{t} y_{2, \alpha}(s) d s, \quad 0 \leq t \leq 1, \\
y_{2, \alpha}^{\prime \prime}(t)=(\alpha-1)+\int_{0}^{t} y_{1, \alpha}(s) d s, \quad 0 \leq t \leq 1, \\
y_{1, \alpha}(0)=\alpha-1, \quad y_{2, \alpha}(0)=1-\alpha, \\
y_{1, \alpha}^{\prime}(0)=0, \quad y_{2, \alpha}^{\prime}(0)=0 .
\end{array}\right\}
$$

The corresponding $(1,1)$-system has the exact solution

$$
\begin{aligned}
y(t)= & {\left[(\alpha-1) e^{0.5 t}\left(\cos \left(\frac{t}{\sqrt{2}}\right)-\frac{1}{\sqrt{2}} \sin \left(\frac{t}{\sqrt{2}}\right)\right),\right.} \\
& \left.(1-\alpha) e^{0.5 t}\left(\cos \left(\frac{t}{\sqrt{2}}\right)-\frac{1}{\sqrt{2}} \sin \left(\frac{t}{\sqrt{2}}\right)\right)\right],
\end{aligned}
$$

and the corresponding $(1,2)$-system has the exact solution

$$
\begin{aligned}
y(t)= & {\left[(\alpha-1) e^{0.5 t}\left(\cos \left(\frac{\sqrt{3}}{2} t\right)-\frac{1}{\sqrt{3}} \sin \left(\frac{\sqrt{3}}{2} t\right)\right),\right.} \\
& \left.(1-\alpha) e^{0.5 t}\left(\cos \left(\frac{\sqrt{3}}{2} t\right)-\frac{1}{\sqrt{3}} \sin \left(\frac{\sqrt{3}}{2} t\right)\right)\right] .
\end{aligned}
$$

Using the RKHS method, taking $\alpha=0.2(i-1), i=1,2, \ldots, 6$, the numerical results at $t=$ 0.5 for $n=10$ are presented for both the $(1,1)$-system and the $(1,2)$-system in Tables 1 and 2 , respectively. In order to show the efficiency of the RKHS method in solving FVIDE (12)(13), absolute errors are calculated at $\alpha=0.5, n=10$ and various $t$ for the two branches of the $(1,1)$-system in Tables 3 and 4 and for the two branches of the $(1,2)$-system in Tables 5 and 6.

Without losing the general issue, we will solve the next two examples, unlike Example 5.1 , either using the $(1,1)$-system or the $(1,2)$-system to avoid repetitions. 
Table 3 The absolute errors of approximating $y_{1, \alpha}$ for $(1,1)$-system at various $t$ and $\alpha=0.5$

\begin{tabular}{llll}
\hline$t_{i}$ & Exact solution & Approximate solution & Absolute error \\
\hline 0 & -0.5 & -0.5 & 0 \\
0.2 & -0.4919942732092149 & -0.49155676396105286 & $4.3750924816210457 \times 10^{-4}$ \\
0.4 & -0.4659175034022215 & -0.46513632990261633 & $7.811734996051589 \times 10^{-4}$ \\
0.6 & -0.4186325706793184 & -0.4177266247210882 & $9.059459582302365 \times 10^{-4}$ \\
0.8 & -0.3470103621084155 & -0.34632653813195646 & $6.838239764590526 \times 10^{-4}$ \\
1 & -0.2480352042594187 & -0.24803520425941872 & 0 \\
\hline
\end{tabular}

Table 4 The absolute errors of approximating $y_{2, \alpha}$ for $(1,1)$-system at various $t$ and $\alpha=0.5$

\begin{tabular}{llll}
\hline$t_{i}$ & Exact solution & Approximate solution & Absolute error \\
\hline 0 & 0.5 & 0.5 & 0 \\
0.2 & 0.4919942732092149 & 0.49155676396105263 & $4.375092481623266 \times 10^{-4}$ \\
0.4 & 0.4659175034022215 & 0.46513632990261655 & $7.811734996049369 \times 10^{-4}$ \\
0.6 & 0.4186325706793184 & 0.41772662472108835 & $9.059459582300699 \times 10^{-4}$ \\
0.8 & 0.3470103621084155 & 0.34632653813195679 & $6.838239764587195 \times 10^{-4}$ \\
1 & 0.2480352042594187 & 0.24803520425941916 & $4.440892098500626 \times 10^{-16}$ \\
\hline
\end{tabular}

Table 5 The absolute errors of approximating $y_{1, \alpha}$ for $(1,2)$-system at various $t$ and $\alpha=0.5$

\begin{tabular}{llll}
\hline$t_{i}$ & Exact solution & Approximate solution & Absolute error \\
\hline 0 & -0.5 & -0.5000000000000001 & $1.1102230246251565 \times 10^{-16}$ \\
0.2 & -0.48933477110786599 & -0.4887536023044621 & $5.811087741978138 \times 10^{-4}$ \\
0.4 & -0.45471216929013725 & -0.45368890889731417 & $1.0232603928230777 \times 10^{-3}$ \\
0.6 & -0.3923561778762831 & -0.3911974694888205 & $1.1587083874625703 \times 10^{-3}$ \\
0.8 & -0.298878446816582 & -0.2980336644678224 & $8.447823487595651 \times 10^{-4}$ \\
1 & -0.1715140126819416 & -0.17151401268194177 & $1.6653345369377348 \times 10^{-16}$ \\
\hline
\end{tabular}

Table 6 The absolute errors of approximating $y_{2, \alpha}$ for $(1,2)$-system at various $t$ and $\alpha=0.5$

\begin{tabular}{llll}
\hline$t_{i}$ & Exact solution & Approximate solution & Absolute error \\
\hline 0 & 0.5 & 0.5000000000000002 & $2.220446049250313 \times 10^{-16}$ \\
0.2 & 0.4893347110786599 & 0.4887536023044618 & $5.811087741980914 \times 10^{-4}$ \\
0.4 & 0.45471216929013725 & 0.45368890889731406 & $1.0232603928231887 \times 10^{-3}$ \\
0.6 & 0.3923561778762831 & 0.3911974694888207 & $1.1587083874623483 \times 10^{-3}$ \\
0.8 & 0.298878446816582 & 0.2980336644678221 & $8.447823487598982 \times 10^{-4}$ \\
1 & 0.1715140126819416 & 0.17151401268194189 & $2.7755575615628914 \times 10^{-16}$ \\
\hline
\end{tabular}

Example 5.2 (see [9]) Consider the following second-order FVIDE:

$$
\begin{aligned}
y^{\prime \prime}(t)= & {\left[(-10+5 \alpha) e^{1-t}-(1+0.5 \alpha) t,(-3-2 \alpha) e^{1-t}-\left(0.6+0.1 \alpha-0.2 \alpha^{2}\right) t\right] } \\
& +\int_{0}^{t}[-0.2+0.1 \alpha,-0.1] e^{s-1} y(s) d s, \quad 0 \leq t \leq 1,
\end{aligned}
$$

subject to the fuzzy initial conditions

$$
y(0)=[(-10+5 \alpha) e,(-3-2 \alpha) e], \quad y^{\prime}(0)=[(3+2 \alpha) e,(10-5 \alpha) e] .
$$


Table 7 The absolute errors of approximating $y_{1, \alpha}$ for $(1,1)$-system at various $t$ and $\alpha$

\begin{tabular}{llllll}
\hline$\alpha$ & $y_{1, \alpha}(0)$ & $y_{1, \alpha}(0.25)$ & $y_{1, \alpha}(0.5)$ & $y_{1, \alpha}(0.75)$ & $y_{1, \alpha}(1)$ \\
\hline 0.5 & $3.55271 \times 10^{-15}$ & $9.35382 \times 10^{-6}$ & $9.70896 \times 10^{-6}$ & $5.66864 \times 10^{-6}$ & $7.10543 \times 10^{-14}$ \\
0.75 & $7.10543 \times 10^{-15}$ & $7.79485 \times 10^{-6}$ & $8.09080 \times 10^{-6}$ & $4.72387 \times 10^{-6}$ & $5.32907 \times 10^{-14}$ \\
1 & $1.24345 \times 10^{-14}$ & $6.23588 \times 10^{-6}$ & $6.47264 \times 10^{-6}$ & $3.77909 \times 10^{-6}$ & $4.61853 \times 10^{-14}$ \\
\hline
\end{tabular}

Table 8 The absolute errors of approximating $y_{2, \alpha}$ for $(1,1)$-system at various $t$ and $\alpha$

\begin{tabular}{llllll}
\hline$\alpha$ & $y_{2, \alpha}(0)$ & $y_{2, \alpha}(0.25)$ & $y_{2, \alpha}(0.5)$ & $y_{2, \alpha}(0.75)$ & $y_{2, \alpha}(1)$ \\
\hline 0.5 & $1.06581 \times 10^{-14}$ & $4.98870 \times 10^{-6}$ & $5.17811 \times 10^{-6}$ & $3.02328 \times 10^{-6}$ & $6.75016 \times 10^{-14}$ \\
0.75 & $1.06581 \times 10^{-14}$ & $5.61229 \times 10^{-6}$ & $5.82538 \times 10^{-6}$ & $3.40119 \times 10^{-6}$ & $4.97379 \times 10^{-14}$ \\
1 & $1.24345 \times 10^{-14}$ & $6.23588 \times 10^{-6}$ & $6.47264 \times 10^{-6}$ & $3.77909 \times 10^{-6}$ & $4.61853 \times 10^{-14}$ \\
\hline
\end{tabular}

Here, the $(1,1)$-system is

$$
\left.\begin{array}{l}
y_{1, \alpha}^{\prime \prime}(t)=(-10+5 \alpha) e^{1-t}-(1+0.5 \alpha) t+\int_{0}^{t}(-0.2+0.1 \alpha) e^{s-1} y_{1, \alpha}(s) d s \\
y_{2, \alpha}^{\prime \prime}(t)=(-3-2 \alpha) e^{1-t}-\left(0.6+0.1 \alpha-0.2 \alpha^{2}\right) t-\int_{0}^{t}(0.1) e^{s-1} y_{2, \alpha}(s) d s, \\
y_{1, \alpha}(0)=(-10+5 \alpha) e, \quad y_{2, \alpha}(0)=(-3-2 \alpha) e \\
y_{1, \alpha}^{\prime}(0)=(3+2 \alpha) e, \quad y_{2, \alpha}^{\prime}(0)=(10-5 \alpha) e
\end{array}\right\}
$$

where $0 \leq t \leq 1$. The corresponding $(1,1)$-system has the exact solution

$$
y(t)=\left[(-10+5 \alpha) e^{1-t},(-3-2 \alpha) e^{1-t}\right]
$$

Using the method presented in Sect. 4, taking $n=100, t_{i}=0.25(i-1), i=1,2, \ldots, 5$, the numerical results of $(1,1)$-system in Example 5.2 in different values of $\alpha$ are shown in Tables 7 and 8 .

Example 5.3 (see [9]) Consider the following second-order FVIDE:

$$
\begin{aligned}
y^{\prime \prime}(t)= & {\left[(3 \alpha-3) \sin t+\frac{3}{20}\left(\alpha^{2}-6 \alpha+5\right) t \cos ^{2} t,\right.} \\
& \left.(9-9 \alpha) \sin t-\frac{9}{20}\left(\alpha^{2}-6 \alpha+5\right) t \cos ^{2} t\right] \\
& +\int_{0}^{t}[0.1+0.3 \alpha, 0.5-0.1 \alpha] t \cos (s) y(s) d s, \quad 0 \leq t \leq 1,
\end{aligned}
$$

subject to the fuzzy initial conditions

$$
y(0)=[0,0], \quad y^{\prime}(0)=[-9+9 \alpha, 3-3 \alpha] .
$$

Here, the $(1,2)$-system is

$$
\begin{aligned}
& y_{2, \alpha}^{\prime \prime}(t)=(3 \alpha-3) \sin t+\frac{3}{20}\left(\alpha^{2}-6 \alpha+5\right) t \cos ^{2} t+\int_{0}^{t}(0.1+0.3 \alpha) t \cos (s) y_{1, \alpha}(s) d s, \\
& y_{1, \alpha}^{\prime \prime}(t)=(9-9 \alpha) \sin t-\frac{9}{20}\left(\alpha^{2}-6 \alpha+5\right) t \cos ^{2} t+\int_{0}^{t}(0.5-0.1 \alpha) t \cos (s) y_{2, \alpha}(s) d s, \\
& y_{1, \alpha}(0)=0, \quad y_{2, \alpha}(0)=0, \\
& y_{1, \alpha}^{\prime}(0)=-9+9 \alpha, \quad y_{2, \alpha}^{\prime}(0)=3-3 \alpha,
\end{aligned}
$$


Table 9 The absolute errors of approximating (1,2)-system at various $t$ and $\alpha=0.5$

\begin{tabular}{rllll}
\hline$n$ & $y_{1, \alpha}(0.16)$ & $y_{2, \alpha}(0.16)$ & $y_{1, \alpha}(0.96)$ & $y_{2, \alpha}(0.96)$ \\
\hline 5 & $4.40853 \times 10^{-3}$ & $1.46951 \times 10^{-3}$ & $1.48218 \times 10^{-2}$ & $4.94061 \times 10^{-3}$ \\
10 & $1.12017 \times 10^{-3}$ & $3.73391 \times 10^{-4}$ & $5.20311 \times 10^{-3}$ & $1.73437 \times 10^{-3}$ \\
100 & $4.84584 \times 10^{-6}$ & $1.61528 \times 10^{-6}$ & $7.23713 \times 10^{-6}$ & $2.41238 \times 10^{-6}$ \\
\hline
\end{tabular}

where $0 \leq t \leq 1$. The corresponding $(1,2)$-system has the exact solution

$$
y(t)=[(-9+9 \alpha) \sin t,(3-3 \alpha) \sin t]
$$

One can observe from Table 9 that as $n$ gets larger, the physical behavior of the absolute errors approaches to zero very rapidly. Anyway, Table 9 also shows that our RKHS method gives reasonable results for small $n$ as well.

\section{Conclusion}

We have introduced a new method for solving second-order FVIDEs based on reproducing kernel theory. The proposed technique gives solutions with good generalization and high exactness. The robustness of this technique has been proved by numerical simulations. As a future research line, this technique can be expanded to solving a class of fuzzy ordinary differential algebraic equations and fuzzy partial integro-differential equations.

Acknowledgements

The authors are grateful to the responsible editor and the anonymous referees for their valuable efforts.

Funding

No funding sources to be declared.

\section{Competing interests}

The authors declare that they have no competing interests.

\section{Authors' contributions}

All the authors worked jointly. All the authors read and approved the final manuscript.

\section{Author details}

${ }^{1}$ Faculty of Engineering Technology, Al-Balqa Applied University, Amman, Jordan. ${ }^{2}$ Department of Applied Science, Ajloun College, Al-Balqa Applied University, Ajloun, Jordan.

\section{Publisher's Note}

Springer Nature remains neutral with regard to jurisdictional claims in published maps and institutional affiliations.

Received: 3 September 2018 Accepted: 16 December 2018 Published online: 29 December 2018

\section{References}

1. Zadeh, L.: Toward a generalized theory of uncertainty (GTU) an outline. Inf. Sci. 175, 1-40 (2005)

2. Alikhani, R., Bahrami, F., Jabbari, A.: Existence of global solutions to nonlinear fuzzy Volterra integro-differential equations. Nonlinear Anal. 75, 1810-1821 (2012)

3. Matinfar, M., Ghanbari, M., Nuraei, R.: Numerical solution of linear fuzzy Volterra integro-differential equations by variational iteration method. J. Intell. Fuzzy Syst. 24, 575-586 (2013)

4. Gumah, G., Moaddy, K., Al-Smadi, M., Hashim, I.: Solutions of uncertain Volterra integral equations by fitted reproducing kernel Hilbert space method. J. Funct. Spaces 2016, Article ID 2920463 (2016)

5. Casasnovas, J., Rossell, F.: Averaging fuzzy biopolymers. Fuzzy Sets Syst. 152, 139-158 (2005)

6. Barros, L.C., Bassanezi, R.C., Tonelli, P.A.: Fuzzy modelling in population dynamics. Ecol. Model. 128, 27-33 (2000)

7. El Naschie, M.S.: From experimental quantum optics to quantum gravity via a fuzzy Kahler manifold. Chaos Solitons Fractals 25, 969-977 (2005)

8. Biswas, S., Roy, T.K.: Generalization of Seikkala derivative and differential transform method for fuzzy Volterra integro-differential equations. J. Intell. Fuzzy Syst. 34, 2795-2806 (2018) 
9. Allahviranloo, T., Khezerloo, M., Sedaghatfar, O., Salahshour, S.: Toward the existence and uniqueness of solutions of second-order fuzzy Volterra integro-differential equations with fuzzy kernel. Neural Comput. Appl. 22(Suppl 1), S133-S141 (2013)

10. Ahmadian, A., Salahshour, S., Chan, C.S., Baleanu, D.: Numerical solutions of fuzzy differential equations by an efficient Runge-Kutta method with generalized differentiability. Fuzzy Sets Syst. 331, 47-67 (2018)

11. Ahmadian, A., Ismail, F., Salahshour, S., Baleanu, D., Ghaemi, F.: Uncertain viscoelastic models with fractional order: a new spectral tau method to study the numerical simulations of the solution. Commun. Nonlinear Sci. Numer. Simul. 53, 44-64 (2017)

12. Saadeh, R., Al-Smadi, M., Gumah, G., Khalil, H., Ali Khan, R.: Numerical investigation for solving two-point fuzzy boundary value problems by reproducing kernel approach. Appl. Math. Inf. Sci. 10(6), 1-13 (2016)

13. Arqub, O.A., Al-Smadi, M., Momani, S., Hayat, T.: Numerical solutions of fuzzy differential equations using reproducing kernel Hilbert space method. Soft Comput. 20, 3283-3302 (2016)

14. Gumah, G., Freihat, A., Al-Smadi, M., Ata, R.B., Ababneh, M.: A reliable computational method for solving first-order periodic BVPs of Fredholm integro-differential equations. Aust. J. Basic Appl. Sci. 8(15), 462-474 (2014)

15. Al-Smadi, M., Arqub, O.A., Shawagfeh, N., Momani, S.: Numerical investigations for systems of second-order periodic boundary value problems using reproducing kernel method. Appl. Math. Comput. 291, 137-148 (2016)

16. Hashemi, M.S., Akgül, A., Inc, M., Mustafa, I.S., Baleanu, D.: Solving the Lane-Emden equation within a reproducing kernel method and group preserving scheme. Mathematics 5(4), 77 (2017)

17. Akgül, A., Karatas, E., Baleanu, D.: Numerical solutions of fractional differential equations of Lane-Emden type by an accurate technique. Adv. Differ. Equ. 2015, 220 (2015)

18. Kaleva, O.: Fuzzy differential equations. Fuzzy Sets Syst. 24, 301-317 (1987)

19. Goetschel, R., Voxman, W.: Elementary fuzzy calculus. Fuzzy Sets Syst. 18, 31-43 (1986)

20. Buckley, J.J., Feuring, T.: Fuzzy differential equations. Fuzzy Sets Syst. 110, 43-54 (2000)

21. Khastan, A., Nieto, J.J.: A boundary value problem for second order fuzzy differential equations. Nonlinear Anal. 72 , 3583-3593 (2010)

22. Mordeson, J., Newman, W.: Fuzzy integral equations. Inf. Sci. 87, 215-229 (1995)

23. Zadeh, L.: Fuzzy sets. Inf. Control 8(3), 338-353 (1965)

24. Geng, F.: A new reproducing kernel Hilbert space method for solving nonlinear fourth-order boundary value problems. Appl. Math. Model. 213, 163-169 (2009)

25. Zhang, S., Liu, L., Diao, L.: Reproducing kernel functions represented by form of polynomials. In: Proceedings of the Second Symposium International Computer Science and Computational Technology, vol. 26, pp. 353-358 (2009)

26. Gumah, G., Naser, M.F.M., Al-Smadi, M., Shawaqfeh, N.: Numerical solutions of hybrid fuzzy differential equations in Hilbert space. Submitted

\section{Submit your manuscript to a SpringerOpen ${ }^{\circ}$ journal and benefit from:}

- Convenient online submission

- Rigorous peer review

- Open access: articles freely available online

- High visibility within the field

- Retaining the copyright to your article

Submit your next manuscript at $\gg$ springeropen.com 